\title{
ÉTUDE DES ÉTOILES SUPERGÉANTES DU GRAND \\ NUAGE DE MAGELLAN, LEURS MAGNITUDES \\ ABSOLUES ET COULEURS
}

\author{
CH. FEHRENBACH \\ Observatoire de Haute-Provence, St. Michel l'Observatoire, 04300 Forcalquier, France
}

\begin{abstract}
Nous indiquons une liste de 29 étoiles membres du Grand Nuage de Magellan que nous proposons comme standards de type spectral des Supergéantes. La situation de ces étoiles dans les diagrammes RH et couleur-couleur est indiquée. Nous proposons des valeurs de $(U-B)_{0}$ et $(B-V)_{0}$ pour ces étoiles.
\end{abstract}

La mesure des vitesses radiales au Prisme Objectif nous a permis de publier en 1970 une liste de 500 étoiles brillantes membres du Grand Nuage et un peu plus de 2000 étoiles de même magnitude apparente de la Galaxie et situées dans la région du Grand Nuage. Depuis cette publication, une centaine d'autres étoiles ont été ajoutées (Fehrenbach et Duflot, 1973).

Une étude systématique de ces étoiles au spectrographe à fente nous a paru très utile, d'autant plus que de nombreuses étoiles du Nuage paraissent beaucoup plus lumineuses que les Supergéantes de la Galaxie. E. Maurice, L. Prévot et moi-même avons pris de nombreux spectres à $73 \AA \mathrm{mm}^{-1}$ et J. P. Brunet et A. Ardeberg ont fait de nombreuses mesures photoélectriques $U B V$. Le catalogue ainsi obtenu sera décrit par Maurice (1972).

Je ne donne ici que la description des classifications spectrales des étoiles et leurs mesures photométriques dans le système $U B V$.

(a) Le diagramme HR ne montre pas dans la représentation $V, B-V$ des classes très marquées d'étoiles. La classification de Morgan et Keenan en classes Ia, Iab, Ib paraît donc à priori difficile.

(b) Nous avons essayé de classer ces étoiles en nous servant soit de standards MK, soit des étoiles classées par les Astronomes de Radcliffe Observatory (Feast et al., 1960). Ceci nous a permis d'établir une classification provisoire en nous servant des critères classiques.

(c) Mais la difficulté provient du fait qu'il n'y a dans le Grand Nuage aucune étoile de type spectral déterminé entre A3 et F8, nous avons donc dû interpoler les types spectraux entre A3 et F8. Ceci nous a amené à proposer une liste (Tableau I) de 29 étoiles standards dont les types sont compris entre O6 et G8. La Figure 1 montre la place des 29 étoiles dans le diagramme HR. La position de nos étoiles dans ce diagramme provient d'un effet de sélection.

Ardeberg et al. (1972) publieront les spectres de ces 29 étoiles. Les types spectraux attribués sont en excellent accord avec ceux de Thackeray pour les 11 étoiles communes. La séquence proposée est donc en accord avec la séquence MK, mais il y a un certain nombre de difficultés. 
TABLEAU I

Liste des étoiles standards proposées

\begin{tabular}{|c|c|c|c|c|c|c|c|}
\hline 1 & & & $V$ & $B-V$ & $U-B$ & Radcliffe & \\
\hline 1 & G 355 & O6f & 12.27 & -0.22 & -1.01 & $\mathrm{R} 115$ & O5f \\
\hline 2 & G 84 & O9f & 11.95 & -0.21 & -0.97 & - & \\
\hline 3 & G 414 & O9.5 I & 11.36 & 0.00 & -0.86 & R129 & B0Ia: \\
\hline 4 & G 420 & O9.5:I & 11.23 & -0.02 & -0.81 & - & \\
\hline 5 & G 22 & B0Ia & 11.34 & -0.12 & -0.95 & R53 & B0Ia: \\
\hline 6 & G 481 & B1Ia & 11.59 & -0.06 & -0.88 & $\mathrm{R} 155$ & B1.5Ia \\
\hline 7 & G 434 & B2Ia & 11.21 & +0.04 & -0.74 & $\mathrm{R} 152$ & B2.5Ia \\
\hline 8 & G 384 & B3Ia & 11.04 & -0.01 & -0.73 & - & \\
\hline 9 & G 283 & B5Ia & 11.59 & -0.01 & -0.69 & R 100 & B3I \\
\hline 10 & G 486 & B5Ia & 11.73 & -0.04 & -0.67 & - & \\
\hline 11 & G 305 & B6Ia & 10.88 & +0.00 & -0.67 & - & \\
\hline 12 & C 10 & B8Ia & (10.95) & $(-0.01)$ & - & R68 & B8I \\
\hline 13 & G 64 & B9Ia & 12.52 & +0.10 & -0.41 & - & \\
\hline 14 & G 390 & A0IaO: & 10.81 & +0.12 & -0.37 & R119 & $\mathrm{A} 0 \mathrm{IaO}$ \\
\hline 15 & G 268 & A0Ia & 10.52 & +0.13 & -0.45 & R98 & A0IaO: \\
\hline 16 & G 321 & $\mathrm{~A} 1 \mathrm{IaO}$ & 10.74 & +0.13 & -0.22 & - & \\
\hline 17 & G 339 & A2IaO: & 10.73 & +0.19 & -0.18 & - & \\
\hline 18 & G 437 & A2I & 11.70 & +0.10 & -0.09 & - & \\
\hline 19 & G 200 & $\mathrm{~A} 3 \mathrm{IaO}$ & 9.12 & +0.19 & -0.24 & R76 & $\mathrm{A} 3: \mathrm{IaO}(\mathrm{e})$ \\
\hline 20 & G 377 & A5Ia: & 11.71 & +0.16 & +0.15 & - & \\
\hline 21 & G 303 & A8: IaO & 10.65 & +0.38 & 0.00 & - & \\
\hline 22 & G 322 & A9Ia & 11.44 & +0.27 & -0.02 & - & \\
\hline 23 & G 247 & F2Ia & 11.20 & +0.27 & +0.10 & - & \\
\hline 24 & G 352 & F6Ia & 10.28 & +0.41 & +0.30 & - & \\
\hline 25 & G 296 & F6Ia & 9.90 & +0.42 & -0.10 & - & \\
\hline 26 & G 460 & F8Ia & 10.20 & +0.68 & +0.50 & - & \\
\hline 27 & G 454 & GOIa & 10.38 & +0.95 & +0.65 & - & \\
\hline 28 & G 367 & G2Ia & 9.91 & +1.15 & +0.60 & $\mathrm{R} 117$ & G0Ia \\
\hline 29 & C 3 & G8Ia & 10.09 & +1.55 & +1.29 & - & \\
\hline
\end{tabular}

(1) La classification visuelle n'est pas suffisante pour attribuer une classe de luminosité et l'accord avec Thackeray n'est pas parfait.

\begin{tabular}{|c|c|c|c|c|}
\hline & \multicolumn{3}{|c|}{ OM } \\
\hline & & $\mathrm{IaO}$ & $\overline{\mathrm{Ia}}$ & $\bar{I}$ \\
\hline \multirow{3}{*}{ Rad. } & $\mathrm{IaO}$ & 2 & 1 & \\
\hline & $\mathrm{Ia}$ & & 4 & $\overline{2}$ \\
\hline & I & & 1 & \\
\hline
\end{tabular}

Et, fait plus grave, il n'y a pas une corrélation nette entre la magnitude absolue et la classe de luminosité. Par classification visuelle, nous obtenons ainsi pour deux étoiles:

$$
\begin{array}{ll}
\text { A0Ia } & V=10.52 \\
\text { A0IaO } & V=10.74 .
\end{array}
$$

(2) Le diagramme HR montre qu'il y a continuité entre les classes de luminosité. Il vaut mieux remplacer les classes $\mathrm{IaO}, \mathrm{Iab}, \mathrm{Ib}$ par des indications directes de la 


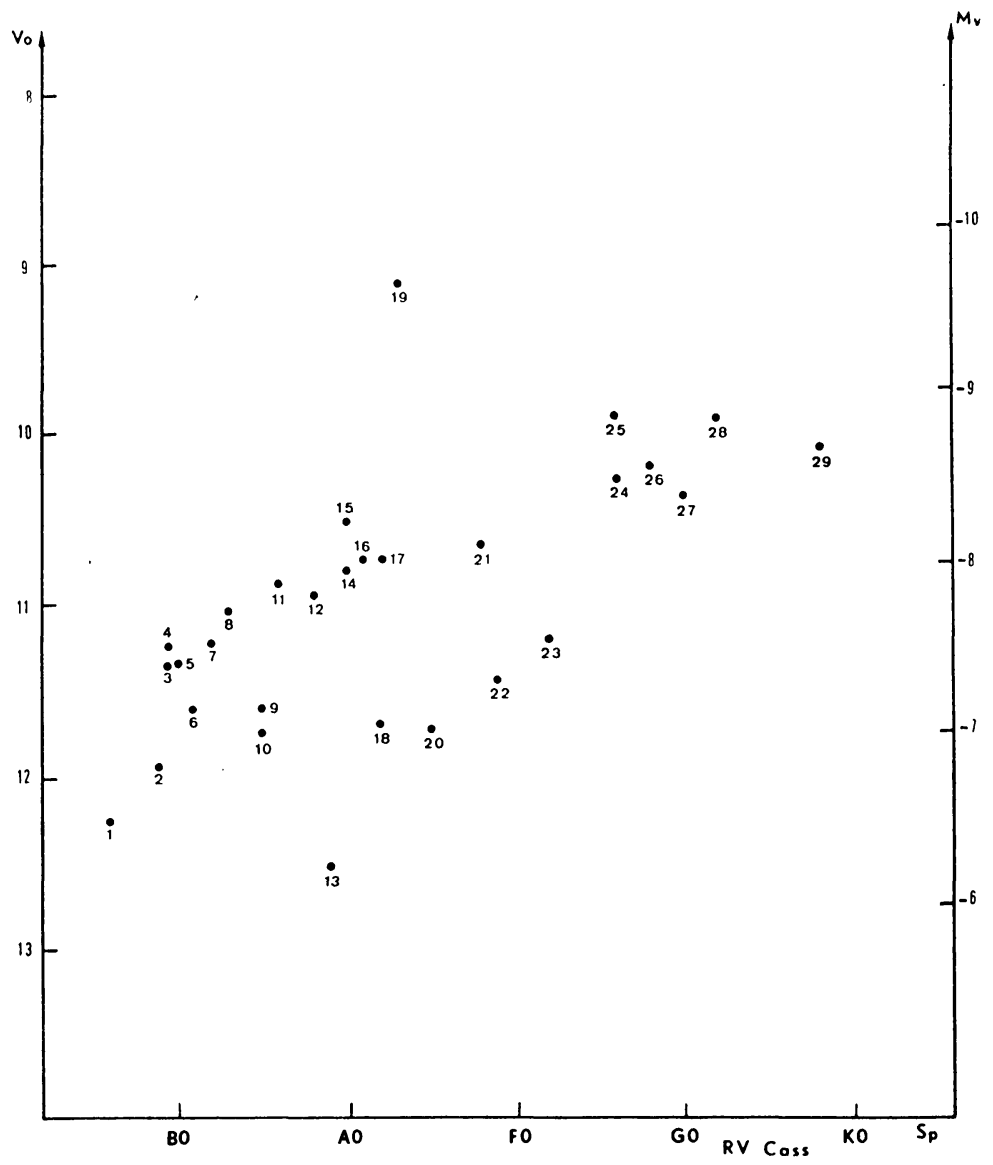

Fig. 1.

magnitude absolue. Il semble que pour les étoiles chaudes la mesure des valeurs de $W \lambda$ pour $\mathrm{H} \gamma$ et $\mathrm{H} \delta$ serait un excellent critère à l'intérieur de chaque classe spectrale. Ces mesures sont en cours (Maurice). Un critère analogue doit être utilisé pour les étoiles avancées.

(3) Il n'est pas possible de donner la magnitude absolue $V$ en fonction du type spectral. Seule une étude quantitative le permettra. Par contre, les couleurs intrinsèques $(B-V)_{0}$ de ces étoiles sont bien déterminées (Figure 2). Les corrections de rougissement suivantes ont été appliquées.

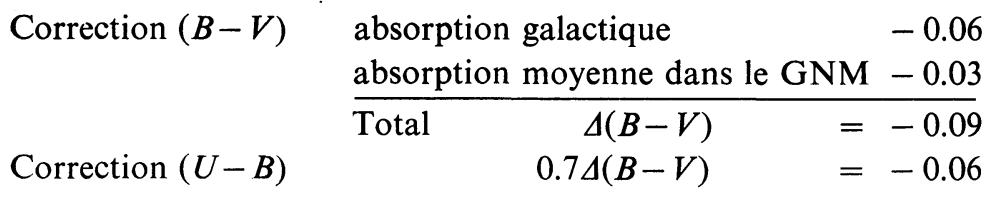

En fait, on constate une différence systématique entre les valeurs de $(B-V)$ pour les étoiles de même type spectral mais de luminosités différentes. 
Pour les étoiles plus brillantes que $m_{v}=10.8$, c'est à dire les supergéantes pour lesquelles $M_{v}<-8$ l'indice de couleur est plus grand de 0.07 que pour les étoiles plus faibles.

$$
\begin{aligned}
& (B-V)=(B-V)+0.07 \\
& M<-8 \quad M>-8 .
\end{aligned}
$$

Les différences $\Delta(B-V)$ ont été déterminées pour l'ensemble des valeurs du catalogue Ardeberg et al. (1972) (Tableau II). Le raccord avec les étoiles III et V sera publié prochainement.

Pour les indices $(U-B)$, la situation est moins nette.

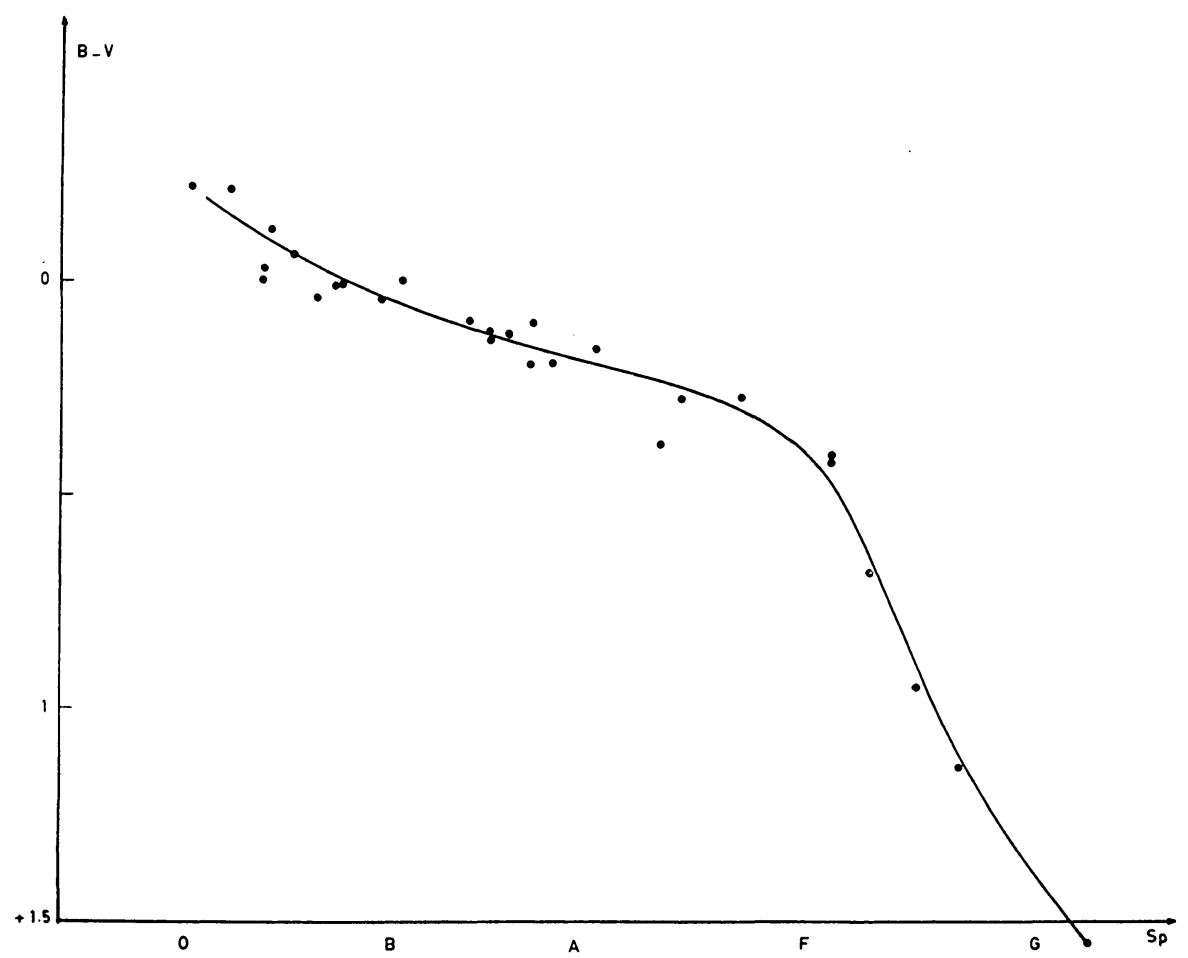

Fig. 2. Position des étoiles standards dans le diagramme spectre-couleur $(B-V)$.

(a) L'écart est dans le sens opposé et varie avec le type spectral, il est de l'ordre de -0.35 pour les étoiles A5. Mais la dispersion des valeurs est considérable.

(b) Il existe des étoiles F6 ayant sensiblement la même valeur de $(B-V)$ et pour lesquelles $(U-B)$ varie d'une étoile à l'autre de +0.53 à -0.10 ! Il ne s'agit pas d'erreurs de mesures car une étude spectrophotométrique de L. Divan donne des résultats pratiquement identiques. Il ne nous semble pas que les étoiles bleues soient situées à l'intérieur de nébuleuses.

La Figure 3 donne la position des étoiles standards dans le diagramme $(U-B)$ $-(B-V)$. Les différences $\Delta(U-B)$ ont été déterminées pour l'ensemble du catalogue Ardeberg et al. (Tableau III). 


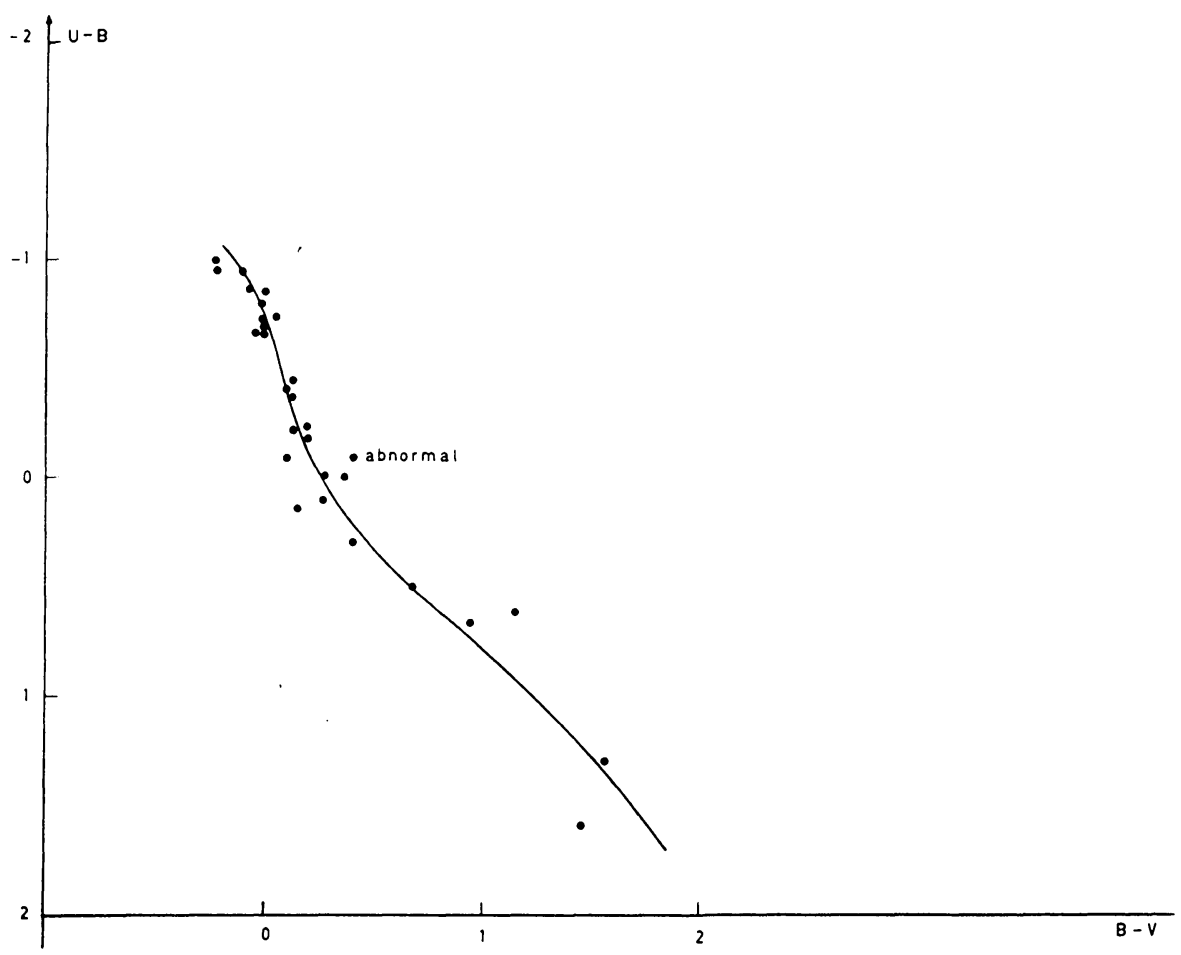

Fig. 3. Position des étoiles standards dans le diagramme couleur-couleur.

TABLEAU II

Valeur de $(B-V)_{0}$ pour les supergéantes du GNM

\begin{tabular}{lccc}
\multicolumn{4}{c}{$(B-V)_{0}$} \\
Sp & $(1)$ & $(2)$ & $\triangle(B-V)_{0}$ \\
& $m<10.8$ & $m>10.8$ & $(1)-(2)$ \\
\hline B0 & -0.15 & -0.22 & +0.07 \\
B5 & -0.05 & -0.13 & 0.08 \\
A0 & 0.03 & -0.03 & 0.06 \\
A5 & 0.15 & +0.08 & 0.07 \\
F0 & 0.27 & 0.19 & 0.08 \\
F5 & 0.42 & 0.37 & 0.05 \\
G0 & 0.80 & & \\
G5 & 1.26 & & \\
& & & \\
\hline
\end{tabular}

\section{Conclusion}

(1) Une mesure de $W \lambda$ de $\mathrm{H} \gamma$ et $\mathrm{H} \delta$ pour les étoiles de spectre $<\mathrm{F} 2$ et la mesure d'un autre indice spectral pour les étoiles $>$ F2 s'impose pour compléter ou remplacer les classes de luminosité MK et ces indices permettront la détermination des magnitudes absolues $M_{v}$. 


\section{TABLEAU III}

Valeur de $(U-B)_{0}$ pour les supergéantes du GNM

$\begin{array}{cccc}\text { Sp } & (U-B)_{0} & & \Delta(U-B) \\ & (1) & (2) & (1)-(2) \\ & m<10.8 & m>10.8 & \\ & & & \\ \text { B0 } & -0.99 & -0.93 & -0.06 \\ \text { B5 } & -0.77 & -0.71 & -0.06 \\ \text { A0 } & -0.53 & -0.38 & -0.15 \\ \text { A5 } & -0.27 & 0.08 & -0.35 \\ \text { F0 } & -0.01 & 0.18 & -0.19 \\ \text { F5 } & 0.24 & 0.21 & -0.03 \\ \text { G0 } & 0.56 & & \\ \text { G5 } & 0.98 & & \\ \text { G8 } & 1.22 & & \end{array}$

(2) Les valeurs de $(B-V)(U-V)_{0}$ sont bien définies et permettent la détermination des excès de couleurs $\mathrm{E}$. Lorsque les mesures de $W \lambda$ seront faites, cet ensemble homogène permettra la détermination des distances galactiques.

(3) Les indices $(U-B)_{0}$ montrent une forte dispersion, néanmoins le diagramme couleur-couleur est utile pour l'étude d'une population stellaire.

\section{Bibliographie}

Ardeberg, A., Brunet, J.P., Maurice, E., et Prévot, L.: 1972, Astron. Astrophys. Suppl. Ser. 6, 249. Fehrenbach, Ch. et Duflot, M.: 1970, Astron. Astrophys., Special Supplement serie No. 1.

Fehrenbach, Ch. et Duflot, M.: 1973, preprint.

Feast, M. W., Thackeray, A. D., et Wesselink, A. J.: 1960, Monthly Notices Roy. Astron. Soc. 121, 337. Maurice, E.: 1972, Communication faite au cours de ce symposium. 